\title{
Comparación entre dos escuelas de Biblioteconomía de Brasil y España: aspectos curriculares
}

\author{
Vânia Martins Bueno de Oliveira Funaro \\ Claudio Marcondes de Castro Filho \\ Facultad de Biblioteconomia e Ciência da Informação \\ Fundación Escuela de Sociologia y Política de São Paulo (Brasil)
}

\subsection{Resumen}

Análisis comparativo del plan de estudios de los cursos de Biblioteconomía impartidos por la Facultad de Biblioteconomia e Ciência da Información de la Fundación Escuela de Sociologia y Política de São Paulo (Brasil) y de la Diplomatura en Biblioteconomía y Documentación de la Universidad Carlos III (Madrid, España). Tras una presentación histórica de la enseñanza de Biblioteconomía en Brasil y España y de su contexto demográfico y geográfico, se evalúa la carga lectiva, la duración de los cursos, el enfoque de los estudios y su adecuación al mercado de trabajo. Además se analizan las instituciones que los imparten en sus aspectos físicos, su estructura organizacional, las becas y ayudas al estudio y las actividades de sus bibliotecas.

Palabras clave: Enseñanza de la Biblioteconomía. Mercado de trabajo. Evaluación de planes de estudio.

\subsection{Abstract}

Comparative analysis of the courses offered by the Faculdade de Biblioteconomia e Ciência da Informação of the Fundación Escuela de Sociologia y Política de São Paulo (Brasil) and the bachelor degree in Library and Information Science of the Universidad Carlos III (Madrid/Spain). The length, extension and adjustment of the courses to the labour market, and installations, organizational structure, grants policy and library services were evaluated.

Keywords: Librarian education. Labour market. Curricula evaluation.

\section{Introducción}

Este trabajo tiene por objetivo realizar una comparación entre los cursos de Biblioteconomía y Ciencia de la Información de Brasil y España a partir de los 
ejemplos de la Universidad Carlos III de Madrid y la Facultad de Biblioteconomía y Ciencia de la Información de la Fundación Escuela de Sociologia y Política de São Paulo (Brasil) (FESPSP) seleccionados por compartir características semejantes. Se evaluaron los planes de estudio, su adecuación al mercado de trabajo, las posibilidades que ofrecían de prácticas y el trasfondo histórico de cada facultad. Creemos que esta comparación ha sido de gran importancia para un análisis y reflexión sobre la adecuación de los planes de estudio - y, en definitiva, de los futuros profesionales de la información - al mercado de trabajo en la línea de otros estudios comparativos realizados anteriormente y divulgados en congresos y monografías, entre los que se destacan aquí el de Barbalho (1996) - que compara la Universidad de Montreal (Canadá) y la Universidad do Amazonas (Brasil) - y el de Cunha (1999) - que analiza comparativamente la formación de los bibliotecarios en Francia, Canadá, Dinamarca y Brasil.

\section{Historia de la Biblioteconomía}

\subsection{Historia de los cursos de Biblioteconomía en Brasil}

El 13 de mayo de 1811, el príncipe Don Juan VI inauguró la Real Biblioteca de Rio de Janeiro. Ésta fue según Lemos (2000, p. 11) "la primera acción del estado portugueses en el área de las bibliotecas en la colonia, que después se convertiría en metrópoli”. En 1991 se creó un curso de Biblioteconomía dependiente de la Biblioteca Nacional, que fue el segundo curso de este tipo en América Latina tras el organizado por el Consejo de Mujeres de Argentina en 1903 (Castro, 2000). Años después surgió la Escuela de Biblioteconomía y Documentación de la FESPSP.

\subsubsection{Facultad de Biblioteconomía y Ciencia de la Información de la FESPSP}

La historia del curso de Biblioteconomía de la FESPSP comienza en 1935 cuando el municipio de São Paulo creó el Departamento de Cultura, con la incorporación de la Biblioteca Municipal, cuyo jefe era Rubens Borba de Moraes, hombre destacado por sus iniciativas culturales. En 1936 la Escuela de Biblioteconomía estaba en pleno funcionamiento en el edificio de la Escuela de Comercio Álvares Penteado y en el recinto destinado a la Escuela de Sociologia y Política de São Paulo. Formaban parte del currículo las disciplinas de Catalogación y Clasificación. Posteriormente fue agregada la Historia del Libro, cuyo profesor fue precisamente Rubens Borba de Moraes. En 1938, se incluyo la disciplina de Referencia. En dicho año concluyeron el curso 69 bibliotecarios, un número bastante significativo para la época. En 1939 el municipio de São Paulo interrumpe los cursos. En 1940 resurge el curso de Biblioteconomía ya a cargo de la FESPSP, siendo el primero de la ciudad y de todo Brasil. En 1943 se decidió transformarlo en Escuela de Biblioteconomía. Como contaba con el auxilio de

Scire. $9: 1$ (en.-jun. 2003) 113-124. 
la Fundación Rockefeller y la colaboración de la American Library Association, se agregaron algunas disciplinas para homologar el currículo a los de las library schools norteamericanas. En 1947 la escuela fue oficialmente reconocida por el gobierno del estado conforme el decreto $\mathrm{N}^{\circ} .17104$ y reglamentada por el decreto $\mathrm{N}^{\circ} .22833$ de 1953, formalizando así los derechos de los alumnos, el título en Biblioteconomía y estableciendo los requisitos del diploma.

Tras varias reestructuraciones del currículo, en 1977 se sometió al Consejo Federal de Educación una propuesta de modificación que procuraba una mejor adecuación al mercado de trabajo. Además, la Escuela pasó a llamarse Facultad de Biblioteconomía y Documentación. En 1984 el programa se amplió de tres a cuatro años, con la inclusión de nuevas disciplinas, como el Trabajo de Conclusión de Curso, las Prácticas Obligatorias Supervisadas, y otras orientadas a la formación en las nuevas tecnologías. En 1999 paso a llamarse Facultad de Biblioteconomía y Ciencia de la Información, adaptando su nombre a las exigencias del mercado de trabajo. Hoy, la Facultad de Biblioteconomía y Ciencia de la Información de la FESPSP cuenta con treinta y dos profesores, que imparten clases técnicas y culturales, y buscan constantemente las técnicas más recientes y apropiadas, al ritmo de las transformaciones que la palabra escrita va sufriendo a lo largo de los siglos; en definitiva, cambiando con ella para mejor servirla.

\subsection{Historia de la Biblioteconomía en España}

La historia de la educación universitaria en Biblioteconomía y Ciencia de la Información en España es relativamente reciente. Durante muchos años, la educación en este campo estuvo ligada exclusivamente a la administración pública.

Durante los primeros años del siglo XX Cataluña se convirtió en el primer lugar de España en adoptar el nuevo concepto de biblioteca pública como una de las claves de su política cultural. En 1915 se creó una escuela en Barcelona para preparar profesionales, que en la época eran exclusivamente mujeres. Al mismo tiempo el gobierno republicano estimuló el desarrollo de la red nacional de bibliotecas populares. Aunque este programa fue destruido en la guerra civil, la escuela de Cataluña continuó y fue incluso imitada en 1967 por la Universidad de Navarra, que creo una escuela para mujeres, que permaneció en funcionamiento hasta 1977. Después de la guerra, el Consejo Superior de Investigaciones (CSIC) creó en Madrid un programa semejante, que se extendió hasta 1973. Años mas tarde el CSIC organizo el Centro de Información y Documentación Científica (CINDOC).

Con el ingreso de la democracia en España, los nuevos gobernantes empezaron un proceso de reforma de la legislación universitaria. Esa legislación abrió las puertas a nuevos estudios. En 1978 fue establecido un título de pregrado en

Scire. $9: 1$ (en.-jun. 2003) 113-124. 
Biblioteconomía y Ciencia de la Información. Hoy existen catorce escuelas, y ocho de ellas poseen cursos de posgrado. En 1994 la licenciatura fue implantada en la Universidad Carlos III de Madrid (García Marco, 2000).

\subsubsection{La Universidad Carlos III de Madrid}

La Universidad Carlos III de Madrid, creada en el año 1989 nace con el objetivo último de prestar a la sociedad un servicio público eficaz y de calidad, contribuyendo a hacer efectivo el derecho a la educación contemplado en la constitución española. Pretende ofrecer un modelo de educación integral, dentro de un concepto unitario y global de la ciencia y la cultura. La filosofía que desde el principio ha presidido su actuación es promover la formación de personas plenamente responsables y libres, sensibles a los problemas sociales y comprometidas con una idea de progreso basada en la libertad, la justicia y la tolerancia.

\section{Planes de estudios (estructura del curso)}

Los contenidos de los cursos se distribuyen en dos grupos: a) contenidos de formación general, destinados a formar en campos del conocimiento externos a la Biblioteconomía; y b) contenidos de formación especifica, que son nucleares con relación a las identidades profesionales existentes. De carácter propedéutico o no, los contenidos de formación general aportan elementos teóricos que tienen por objetivo mejorar el aprovechamiento de los específicos. Los contenidos específicos o profesionales tienen carácter terminal y constituyen el núcleo básico en el cual se basa la formación de los bibliotecarios. Ciertas materias transversales como la Metodología de la Investigación, las Tecnologías de Información, etc., pueden ser objeto de temas curriculares específicos o de actividades practicadas en el ámbito de otras asignaturas.

Se recomienda que los proyectos académicos acentúen la adopción de una perspectiva humanística en la formulación de los contenidos, confiriéndoles un sentido social y cultural que sobrepase los aspectos utilitarios más inmediatos. Ello supone, recíprocamente, conocer las exigencias que plantean los centros de trabajo a la formación. En el caso de la información y documentación en España, no hay ninguna duda de que la irrupción en el mercado laboral de titulados de todos los ciclos ha tenido unas consecuencias que conviene indagar. Hay que detectar, por una parte, las características que debe poseer la formación de los profesionales de la información para realizar adecuadamente sus tareas laborales de acuerdo a las funciones que les puedan asignar los responsables de las unidades de información; y, por otra, determinar las necesidades de formación que se exigen a un profesional de la información para ingresar en el mercado laboral.

Moreiro y Caridad (1998) mencionan que sería necesario evaluar la excelencia de los programas docentes, pues no en vano una de las misiones de la

Scire. $9: 1$ (en.-jun. 2003) 113-124. 
Universidad es preparar para el ejercicio profesional, lo cual exige capacidad para adaptar el rumbo de la enseñanza que ofrece la Universidad al cambiante mercado de trabajo y a sus necesidades; y ello tanto desde factores internos - cualificación del profesorado, infraestructuras, selección y atención a los alumnos, idoneidad de los planes de estudio... - como externos - comunicación entre los miembros de la universidad, las empresas y los representantes sociales-. Sólo desde los resultados de estudios que contemplen estos tres últimos sectores cabe delinear los modelos de articulación entre universidad, mercado de trabajo y sociedad.

Los planes de estudios de ambas facultades se presentan para su evaluación y comparación en las tablas I y II. A partir de ellas, es posible detectar la existencia de necesidades subyacentes semejantes, aunque existen diferencias interesantes en el tratamiento de materias específicas. Así, en la FaBCI/FESPSP se presta más atención a la indización que en la Universidad Carlos III, mientras que con la catalogación sucede al revés. Las asignaturas de Informática retratan la realidad del mercado de trabajo: en Brasil se enfatiza la formación en la base de datos WinIsis, cuyo lenguaje es de fácil recuperación y acceso para los usuarios. Por su

\begin{tabular}{|l|l|}
\hline Semestres & Asignaturas \\
\hline Primer semestre & $\begin{array}{l}\text { Lengua y Literatura Portuguesa. Teoría de la Comunicación. Lógica. Producción } \\
\text { de los Registros del Conocimiento I. Introducción a la Biblioteconomía y Ciencia } \\
\text { de la Información }\end{array}$ \\
\hline Segundo semestre & $\begin{array}{l}\text { Representación Descriptiva I. Bibliotecas y Unidades de Información. Informáti- } \\
\text { ca. Lenguajes de Indización I. Lengua Inglesa. Psicología Social }\end{array}$ \\
\hline Tercer semestre & $\begin{array}{l}\text { Representación Descriptiva II. Lenguajes de Indización II. Estadistica. Técnicas } \\
\text { de Redacción. Filosofía }\end{array}$ \\
\hline Cuarto semestre & $\begin{array}{l}\text { Fuentes de Información I. Lenguajes de Indización III. Métodos y Técnicas de } \\
\text { Investigación. Formación y Desarrollo de Colecciones. Teoria de la Administra- } \\
\text { ción. Biblioteca Laboratorio }\end{array}$ \\
\hline Quinto semestre & $\begin{array}{l}\text { Fuentes de Información II. Lenguajes de Indización IV. Gestión de Unidades } \\
\text { de Información I. Sociologia. Producción de los Registros del Conocimiento II. } \\
\text { Practica en Biblioteconomía (prácticas) }\end{array}$ \\
\hline Sexto semestre & $\begin{array}{l}\text { Multimedia. Lenguajes de Indización V. Gestión de Unidades de Información II. } \\
\text { Diseminación de la Información. Acción Cultural. Practica en Biblioteconomía } \\
\text { (prácticas) }\end{array}$ \\
\hline Séptimo semestre & $\begin{array}{l}\text { Conservación y Restauración. Automatización de los Sistemas de Información. } \\
\text { Planeamiento de Unidades de Información. Archivos. Trabajo de Conclusión del } \\
\text { Curso }\end{array}$ \\
\hline Octavo semestre & $\begin{array}{l}\text { Edición. Generación de Bases de Datos Winlsis. Realidad del Brasil Contempo- } \\
\text { ráneo. Trabajo de Conclusión del Curso (orientación y práctica) }\end{array}$ \\
\hline
\end{tabular}

Tabla 1. Plan de estudios de la Facultad de Biblioteconomía y Ciencia de la Información/FESPSP

Scire. $9: 1$ (en.-jun. 2003) 113-124. 


\begin{tabular}{|c|c|c|}
\hline Curso & Cuatrimestre & Asignaturas \\
\hline \multirow{3}{*}{ 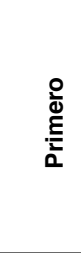 } & $\begin{array}{l}\text { Primer } \\
\text { semestre }\end{array}$ & $\begin{array}{l}\text { Análisis documental I ( } 8 \text { créditos). Fuentes de información generales } \\
\text { ( } 6 \text { créditos). Introducción a la informática ( } 6 \text { créditos). Introducción a } \\
\text { la información ( } 7 \text { créditos) }\end{array}$ \\
\hline & Segundo semestre & $\begin{array}{l}\text { Análisis documental II ( } 7 \text { créditos). Información secundaria: biblio- } \\
\text { grafía ( } 6 \text { créditos). Informática documental ( } 6 \text { créditos) }\end{array}$ \\
\hline & & $\begin{array}{l}\text { Optativa: } 2 \text { ( } 7 \text { créditos): Historia del documento y de las instituciones } \\
\text { documentales. Introducción a la contabilidad. Propiedad intelectual }\end{array}$ \\
\hline \multirow{3}{*}{ 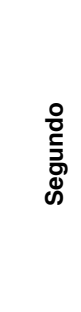 } & $\begin{array}{l}\text { Primer } \\
\text { semestre }\end{array}$ & $\begin{array}{l}\text { Sistemas de clasificación ( } 6 \text { créditos). Archivística general ( } 6 \\
\text { créditos). Tecnología de la información ( } 6 \text { créditos). Fuentes de } \\
\text { información especializadas ( } 7 \text { créditos) }\end{array}$ \\
\hline & Segundo semestre & $\begin{array}{l}\text { Lenguajes documentales ( } 6 \text { créditos). Documentación administrativa } \\
\text { ( } 6 \text { créditos). Administración de bibliotecas y unidades de información } \\
\text { ( } 7 \text { créditos). Prácticas en unidades de información I ( } 6 \text { créditos). } \\
\text { Libre elección: } 1 \text { ( } 7 \text { créditos) }\end{array}$ \\
\hline & & $\begin{array}{l}\text { Optativa: } 1 \text { ( } 6 \text { créditos): Catalogación de materiales especiales. Pu- } \\
\text { blicaciones oficiales y gubernamentales. Introducción a la lingüística }\end{array}$ \\
\hline \multirow{3}{*}{$\frac{\circ}{\frac{0}{0}}$} & $\begin{array}{l}\text { Primer } \\
\text { semestre }\end{array}$ & $\begin{array}{l}\text { Técnicas historiográficas de la investigación documental ( } 6 \text { cré- } \\
\text { ditos). Estudios de usuarios ( } 7 \text { créditos). Gestión de recursos en } \\
\text { bibliotecas y unidades de información ( } 6 \text { créditos). Catalogación } \\
\text { automatizada ( } 7 \text { créditos) }\end{array}$ \\
\hline & Segundo semestre & $\begin{array}{l}\text { Automatización de centros y servicios de información ( } 7 \text { créditos). } \\
\text { Información para la industria ( } 7 \text { créditos). Prácticas en unidades de } \\
\text { información II ( } 6 \text { créditos). Libre elección: } 2 \text { ( } \& \text { créditos) }\end{array}$ \\
\hline & & $\begin{array}{l}\text { Optativa: } 1 \text { ( } 7 \text { créditos): Historia de la administración pública. Régi- } \\
\text { men jurídico de la documentación. Análisis de sistemas de gestión } \\
\text { documental }\end{array}$ \\
\hline * & & $\begin{array}{l}\text { Créditos no asignados a curso: Humanidades (6 créditos). Inglés } \\
\text { (Prueba de nivel I: } 6 \text { créditos) }\end{array}$ \\
\hline
\end{tabular}

Tabla 2. Plan de estudios de la Universidad Carlos III de Madrid

parte, la Universidad Carlos III completa su currículo con disciplinas optativas de gran interés para el mercado de trabajo, mientras que la FaBCI/FESPSP posee solo disciplinas obligatorias. Finalmente, destaca en la facultad brasileña la exigencia de un trabajo de Conclusión del Curso (TCC) como prerrequisito para la obtención del título, que consiste en una monografía cuyo tema es acordado entre el alumno y el coordinador de la disciplina.

\subsection{Practicas y actividades complementarias}

Las prácticas son un mecanismo fundamental de interacción del alumno con el mundo de trabajo en su área. Deben realizarse dentro de los programas académicos de las asignaturas, con intensidad variable dependiendo de la naturaleza de las materias, bajo la responsabilidad de cada docente. Estas prácticas constituyen instrumentos privilegiados para asociar desempeño y contenido de

Scire. $9: 1$ (en.-jun. 2003) 113-124. 
forma sistemática y permanente. Además de eso, los alumnos del curso pueden tener la oportunidad de realizar actividades complementarias de monitorización, investigación, participación en seminarios y congresos, realización de visitas programadas y de otras actividades académicas y culturales, bajo la orientación de (preferentemente en régimen de tutoría) y computables en su carga horaria.

La Universidad Carlos III presta una especial atención y apoyo a la incorporación de sus titulados al mercado de trabajo, promoviendo el contacto entre el mundo de la empresa y los estudiantes a través de las prácticas profesionales en empresas o instituciones públicas en el último año de estudio y a través del Servicio de Orientación y Planificación Profesional (SOPP). Esta unidad, gestionada por la Fundación Universidad Carlos III, presta a los alumnos servicios de información, de orientación, bolsa de empleo, etc. En el mercado de trabajo de Biblioteconomía en Brasil la oferta de practicas en empresas privadas y públicas, en los diversos sectores de la sociedad, ha aumentado considerablemente y favorece la conversión en práctica de lo aprendido en teoría durante el curso. Podemos decir, pues, que las realidades española y brasileña son semejantes.

\section{Perfil profesional}

La formación del bibliotecario supone el desarrollo de determinadas competencias y habilidades y el dominio de los contenidos de la Biblioteconomía. Además de prepararlos para enfrentar con eficiencia y creatividad los problemas de la práctica profesional, producir conocimientos, actuar críticamente sobre la realidad que los envuelve, buscar el perfeccionamiento continuo y observar normas éticas de conducta, los egresados deberán ser capaces de actuar eficazmente en el seno de las instituciones y servicios que demanden sus servicios - bibliotecas, centros de documentación o información, centros culturales, servicios o redes de información, órganos de gestión de patrimonio cultural, etc. - . Las instituciones de Enseñanza Superior podrán facilitar la especialización del egresado en los proyectos académicos y en la organización curricular, sin perjudicar el nivel mínimo aquí considerado.

\subsection{Competencias y habilidades}

Las competencias y habilidades de los graduados en biblioteconomía se pueden dividir en generales y específicas. Entre las generales destacan generar productos a partir de los conocimientos adquiridos y divulgados; formular y ejecutar políticas institucionales; elaborar, coordinar, ejecutar y evaluar planes, programas y proyectos; utilizar racionalmente los recursos disponibles; desarrollar o utilizar nuevas tecnologías; traducir las necesidades de individuos, grupos y comunidades en las respectivas áreas de actuación; desarrollar actividades profesionales autónomas - a saber, orientar, dirigir, asesorar, prestar consulto-

Scire. $9: 1$ (en.-jun. 2003) 113-124. 
ría, realizar peritajes y emitir informes técnicos -; y responder a las demandas sociales de información producidas por las transformaciones tecnológicas que caracterizan al mundo contemporáneo. Entre las específicas están interactuar y agregar valor en los procesos de generación, transferencia y uso de la información en cualquier ambiente; criticar, investigar, proponer, planear, ejecutar y evaluar recursos y productos de información de cualquier naturaleza; procesar la información registrada en diferentes tipos de soporte, mediante la aplicación de conocimientos teóricos y prácticos de colecta, procesamiento, almacenamiento y difusión de la información; y realizar investigaciones relativas a productos, procesamiento y transferencia y uso de información.

\subsection{Facultad de Biblioteconomía y Ciencia de la Informação/FESPSP}

La Biblioteconomía es una de las profesiones que más se han desarrollado en los últimos tiempos. El bibliotecario, hoy en día, ejerce actividades en agencias de publicidad, despachos de abogados, editoras, periódicos, revistas, productoras de video, universidades, bancos, museos, archivos públicos y privados, escuelas de idiomas, empresas de ingeniería, centros de información, y presta asesoría a empresas particulares. Gracias a este amplísimo abanico de campos de actuación, la Biblioteconomía ha pasado a ser una profesión de actualidad, ya que esta implicada en los procesos de globalización, de informatización por medio de las nuevas tecnologías y en la divulgación de datos para un mercado en constante transformación. Actualmente, la información es una de las herramientas más importantes para el desarrollo de un país. Al lado de las materias primas, las inversiones y la mano de obra calificada, la información es generadora de riqueza y de crecimiento económico. Hoy, estar bien informado es un requisito básico para ejercer la ciudadanía y para hacer uso de todas las potencialidades que la tecnología moderna coloca a nuestro alcance. Por otro lado, el volumen de datos que nos sobrecarga todos los días impone la necesidad de organizarlos, seleccionarlos, hacerlos útiles y apropiados para ser transformados en conocimiento. Es allí donde entran en acción los bibliotecarios, unos profesionales cada vez mas reconocidos como administradores de los archivos de información — gestores de la información -, pues les toca administrarla, procesarla y eliminarla, utilizando desde los medios más tradicionales hasta los mas avanzados, como la Internet. Como se señalaba anteriormente, buscando constantemente las técnicas más recientes y apropiadas, los bibliotecarios acompañan las transformaciones que la palabra escrita va sufriendo a lo largo de los siglos, y para mejor servirla, cambian con ella.

\subsection{Universidad Carlos III de Madrid}

La Diplomatura en Biblioteconomía y Documentación se ocupa de formar profesionales con los conocimientos y destrezas fundamentales de la

Scire. $9: 1$ (en.-jun. 2003) 113-124. 
Biblioteconomía, Documentación y Archivística, para gestionar la información en cualquier centro o servicio que se encargue de su tratamiento, como las bibliotecas, generales o especializadas, los archivos de la administración o las empresas y los centros de documentación, así como los de los medios informativos o los centros de investigación. Para ello ofrece una formación completa y coherente, tanto teórica como práctica, a unos profesionales que han de enfrentarse a la nueva Sociedad de la Información, en un contexto tecnológico y social vertiginosamente cambiante, en el que estos profesionales de la información tienen un papel fundamental. En este sentido, la titulación va ahondando cada vez más en la integración de las Tecnologías de la Información y las Comunicaciones en los programas de aquellas asignaturas en las que son aplicables en la realización de actividades directamente vinculadas con su contenido. Además, dos meses de prácticas en unidades de información reales del sector público o privado acercan al alumno al mundo profesional. Al terminar sus estudios, además de encontrar ofertas de trabajo de empresas o de la administración, podrá concursar a las oposiciones específicas para bibliotecarios o archiveros convocadas por las diversas administraciones públicas. Los diplomados en Biblioteconomía y Documentación que deseen conseguir un título superior universitario en el misma área de conocimiento pueden continuar sus estudios cursando, sin necesidad de complementos de formación, la Licenciatura en Documentación.

\section{El mercado de trabajo del profesional de la información}

Los nuevos rumbos de la Biblioteconomía y los cambios en el mercado de trabajo han incrementado las exigencias hacia los profesionales de la información y el mercado de trabajo selecciona profesionales más cualificados. Souza (1996, p. 160) en su investigación sobre el mercado de trabajo del bibliotecario detectó que los requisitos más exigidos para los empleos son experiencia previa, conocimientos de lenguas extranjeras, conocimientos de Informática y titulación. Actualmente -o sea, cinco años después de esta investigación - podemos afirmar que la Informática, las áreas especificas de trabajo y el inglés continúan siendo necesarios para un mejor desempeño en el mercado de trabajo. Las tecnologías vigentes exigen cada vez más que el profesional de la información sea específico en su área de trabajo y al mismo tiempo cualificado. Además, se están produciendo cambios en diversos segmentos de la sociedad que están provocando procesos de adaptación en el mercado de trabajo, incluyendo la aparición de nuevas modalidades de trabajo, como los profesionales en ejercicio libre.

Con el desarrollo tecnológico algunas profesiones están en fase de ebullición y otras quedando obsoletas. La competitividad entre las profesiones se está incrementado en la media en que el cliente es más exigente con la calidad de los servicios. Las nuevas tecnologías dan al profesional de la información nuevas

Scire. $9: 1$ (en.-jun. 2003) 113-124. 
perspectivas en el mercado de trabajo para abordar la evolución de la información. Pero, para ello, este profesional necesita de características específicas entre las que destacan el conocimiento de la información, la facilidad de comunicación, conocimientos técnicos y cultura general elevados, experiencia profesional, espíritu de liderazgo, habilidad de trabajar en equipos multidisciplinares, habilidad para la racionalización de procedimientos, datos y documentos, conocimientos de Informática, visión estratégica y logística de los negocios, capacidad de planear a corto, medio y largo plazo, y capacidad para absorber rápidamente nuevos conceptos en el ambiente profesional (Modesto, 1998, p. 2).

Analizando algunas de estas características podemos concluir que el profesional de la información trabaja con la información y con la búsqueda del conocimiento de otros y, por lo tanto, su conocimiento es de suma importancia para conseguir los objetivos planeados. Por otra lado, la comunicación es el eje de conexión entre el cliente y la información deseada, y la necesidad de investigar el objeto deseado requiere de facilidad de comunicación. Por ello, el grado de escolaridad debe ser de alto nivel, procurando siempre conseguir con plenitud los conocimientos en los diversos segmentos de su profesión. Según Carvalho y Tavares (2001, p. 45).

El conocimiento es una característica del ser humano, que es el único ser capaz de entrar en contacto con una determinada realidad y extraer de ella las informaciones que le son necesarias para la formación de un nuevo entendimiento o nuevo juicio de valor, y aun, es el único que posee la capacidad de aprender, a partir de un cuestionamiento de la realidad, y de transmitir su aprendizaje a sus pares.

La experiencia profesional es un punto esencial para su propio desarrollo, ya que actualmente en el mercado de trabajo constituye el factor de mayor importancia para afirmarse en la sociedad. La experiencia es el retrato del profesional, es su capital intelectual, es la validación de su conocimiento, que le dará la oportunidad de crecimiento en el mercado globalizado. Además, actualmente en las grandes corporaciones el profesional de la información requiere espíritu de líder, pues la transmisión, almacenamiento y expurgo de la información - que son sus responsabilidades - exigen una dinámica de trabajo en grupo, que, como tal, requiere liderazgo. Es especialmente en el proceso de eliminar la información donde el profesional de la información pasa a tener más necesidad de trabajar con equipos multidisciplinares y ocuparse de conectar ideas y personas, desarrollando servicios, sistemas, organización y métodos, en definitiva planificando e implementando soluciones. El profesional de la información tecnologizado necesita dirigir sus esfuerzos a la racionalización de los procedimientos de información, en los que la principal tarea es localizar la información, encontrarla, y no tanto preocuparse de obtenerla como un objeto precioso.

Con la demanda de información rápida en los negocios y la industria, ha surgido espacio para actividades alternativas encuadradas en un nuevo paradigma, 
basado en el enfoque de servicios. En este ambiente de negocios, es necesario contar con investigadores con experiencia, que utilicen las nuevas tecnología digitales con la intención de recuperar la información de forma exhaustiva y precisa. En este sentido Vergueiro (1997) relata:

En una época marcada por los cambios rápidos y avasalladores. De ideas. De perspectivas. De posibilidades. De tecnologías que surgen y se agitan casi en un abrir y cerrar de ojos. De cambios que, específicamente en el área de la información, ocurrieron con una rapidez aun más espantosa, evidenciando por ellas mismas un pasado que parece apenas corroborar previsiones apocalípticas.

La capacidad de los profesionales de la información de absorber nuevos conceptos está ligada a su conocimiento de la tecnología y la información. Según Cunha (2000, p. 160) "el trato con la información en la sociedad contemporánea requiere de la actuación de profesionales con una gran claridad de competencias". Está claro que están surgiendo nuevos conceptos y, consecuentemente, que son esenciales para el profesional de la información la diversificación de actividades, una mayor calidad en el trabajo, mayor profesionalidad y una competencia notable en la interrelación de informaciones diversas.

El horizonte es esperanzador y parece que se están dando los pasos correctos en el ámbito educativo. Así, en España, la Diplomatura en Biblioteconomía y Documentación ha experimentado en los últimos años una mejora notable en la tasa de inserción laboral, llegando con la promoción de 1998 a superar la media española. El 81,6\% de los titulados estaba ocupado en el momento de la realización de la encuesta, y a los cinco años de finalizar los estudios, el $95 \%$.

\section{Conclusiones}

Este estudio comparativo ha permitido verificar que la carga horaria y la duración de los cursos de ambas facultades son adecuadas, y que sus objetivos y contenidos están alineados con las necesidades del mercado de trabajo. Las materias impartidas están orientadas a atender la demanda de los bibliotecarios, $\mathrm{y}$, en su conjunto, de todos los aspectos relacionados con la organización y administración de los sistemas de información. Actualmente, el mercado de trabajo requiere profesionales calificados en las áreas de indización e Informática debido a la fuerte demanda que se está produciendo en redes de bibliotecas, Internet, elaboración de bases de datos, y bibliotecas digitales y virtuales. Por otra parte, la cualificación de los profesionales depende de la teoría aplicada en las facultades, así como de la posibilidad de hacer prácticas. El campo de actuación está bastante diversificado, como pudo observarse en el apartado 4.2.

En defintiva, la información es una de las herramientas esenciales para el desarrollo de un país junto con las materias primas, las inversiones y la mano de obra calificada. La información es generadora de riqueza y de crecimiento

Scire. $9: 1$ (en.-jun. 2003) 113-124. 
económico. Hoy, estar bien informado es un requisito básico para que se pueda ejercer la ciudadana, así como para conocer y hacer uso de todos los beneficios que la tecnología moderna coloca a nuestro alcance.

\section{Bibliografía}

Barbalho, Celia Regina Simonetti (1996). Gerenciando unidades de informação: a formação profissional. Manaus: Editora da Universidade do Amazonas, 1996.

Carvalho, Gilda Maria Rocha de; Tavares, Márcia da Silva (2001). Informação \& Conhecimento: una abordagem organizacional. Rio de Janeiro: Qualitymark, 2001.

Castro, César Augusto (2000). Historia da biblioteconomía brasileira. http://www.geocites. com/ResearchTriangle/2851/RUBEN3.htm. Consultado: 2002-02-05.

Cunha, Miriam Vieira da (1999). A formação en ciência da informação na França, no Canadá e na Dinamarca: comparação con o sistema brasileira. // Anais do 7o Encontro das Escolas de Biblioteconomía da região Sul: Rio Grande, RS, Jun. 24, 1999. Porto Alegre, RS: ABRS, 1999.

Cunha, Miriam Vieira da (2000). O profissional da informação e o mercado de trabalho. // Informação e Sociedade: estudos. 10:1 (Jan. -Jun. 2000) 159-167.

García Marco, Francisco Javier; Agustin Lacruz, Maria del Carmen (2000). Education the information professional of the 21 st century: a ten-point proposal based on the Spanish context. // Education for Information. 18:2-3 (2000) 141-151.

Inserción laboral del diplomado en biblioteconomía y documentación. URL: http:// www. uc3m.es/uc3m/gral/ES/ESCU/escud51-InsercionLaboral.htmp. Consultado: 2002-04-01.

Lemos, Antonio Agenor Briquet de (2000). Cinqüenta anos de sonhos e esperanças. // Perspectivas en Ciência da Informação. 5 (Jan. -Jun. 2000) 11-15.

Modesto, Fernando (1998). O Bibliotecario e o mercado de trabalho: estratégias para o emprego. São Paulo: APB, 1998. (Ensaios da APB, n. 59).

Moreiro, José A.; Caridad, Mercedes (1998). Acerca de los métodos de estudio de la relación entre las condiciones laborales y formativas en biblioteconomía y documentación: el caso de la Universidad Carlos III. // Anales de Documentación. 1 (1998) 137-153.

Plan de estudios de biblioteconomía y documentación. URL: http://www.uc3m.es/uc3m gral/ES/ESCU/escu.51b.htmp. Consultado: 2002-03-19.

Souza, Marta Alves de. Perfil profissional do bibliotecario no mercado de trabalho da cidade de São Paulo. // Transinformação. 8:1 (Jan. -Abr. 1996) 158-166.

Vergueiro, Waldomiro de Castro (1997). A globalização da informação e o futuro das bibliotecas: a valorização do cliente como alternativa profissional. // Anais do $18 \mathrm{o}$ Congresso Brasileiro de Biblioteconomía e Documentação: São Luis, MA, julho 24, 1997. São Luis, MA: Associação de Bibliotecarios do Maranhão, 1997. 